\title{
Consumer Decision-Making Power Based on BP Neural Network and Fuzzy Mathematical Model
}

\author{
Weijie Li \\ School of Economics \& Management, Ankang University, Ankang, 725000 Shaanxi, China \\ Correspondence should be addressed to Weijie Li; liweijie@aku.edu.cn
}

Received 30 April 2021; Revised 1 July 2021; Accepted 28 July 2021; Published 15 August 2021

Academic Editor: Wenqing Wu

Copyright ( 2021 Weijie Li. This is an open access article distributed under the Creative Commons Attribution License, which permits unrestricted use, distribution, and reproduction in any medium, provided the original work is properly cited.

\begin{abstract}
In real life, because of the uncertainty of risk, incomplete information, perceived cost, and other factors, there are irrational behaviors in the decision-making power of consumers, so it is of great practical significance to study the decision-making power of consumers in the choice of countermeasures and personalized product recommendation. The purpose of this paper is to analyze the decision-making power of consumers based on the BP neural network and fuzzy mathematical model. First, the basic theory of artificial neural network and the concepts of set theory and fuzzy reasoning of fuzzy mathematics are described. Second, the behavior prediction model with the equal emphasis on rationality and irrationality and the integration of artificial neural network and fuzzy mathematics are constructed. The comments of a certain mobile phone are selected as the experimental objects to analyze the decision-making reasoning and prediction of individual consumers in the network and the decision-making reasoning of group consumers in the network, the experimental results show that through Mamdani reasoning, behavioral intention $=5.72$. Through the fuzzy set processing, it is finally determined that the consumer's purchase intention is close to the VT mode, which is "very inclined." In the first method, the user's recognition rate of product $\mathrm{C} 1$ is $82 \%$, and in the second method, the user's recognition rate is 55\%. The comparison of the two methods is in line with the expectation. The first method extracts the user's emotion and evaluation information from the comments, fully considers the personalized needs of consumers, and is closer to the prediction results of the system.
\end{abstract}

\section{Introduction}

With the rapid development of e-commerce, many consumers begin to choose to consume online. Because of the virtual, interactive, convenient, and word-of-mouth communication of online consumption, the role of irrational factors is more prominent. At the same time, people's ability in learning, thinking, and action is limited. Psychology and emotion play a great role in purchase intention and behavior. Therefore, it is necessary to fully consider the irrational emotion in the prediction of online consumers' decision-making power. Online consumers often describe their opinions and feelings about the purchased goods or services through online comments, and emotion is the irrational performance. Therefore, we can fully consider the irrational factors of online consumers by mining the emotional information contained in online reviews, so as to better predict the decisionmaking power of consumers. Both model control technology and artificial neural network belong to artificial intelligence technology. For the latter, learning operation can be carried out, but fuzzy information cannot be described. Therefore, it is necessary to integrate the two and, then, build a corresponding system, so that it can not only master the learning ability but also process the fuzzy information.

At present, the emotional analysis of consumers is just in its infancy, and it is rare to predict the behavior of online consumers by using massive online comments to calculate the emotional and product cognition information of consumers. The combination of emotion calculation and decision-making power prediction of online consumers, supplemented by fuzzy sets and fuzzy reasoning, will produce more important theoretical guidance and application value for the formulation of marketing strategies of merchants. At the same time, the discussion of online consumer group behavior will lead the research of group behavior in an ecommerce environment. The prediction results are of great 
practical significance for the selection of countermeasures and recommendation of personalized products in network marketing and personalized marketing.

In this paper, based on the evaluation system of the decision-making power of online consumers, the fuzzy processing is carried out, and the degree is divided into seven categories from weak to strong. The calculation method based on the dimension of the behavior prediction model is given, and the trial calculation is carried out. According to the behavior prediction model of both rationality and irrationality, the fuzzy reasoning rule base is established with the subjective attitude of online consumers and the subjective norms of online consumption as the antecedent and the decision-making power of consumers as the consequent, which realizes the reasoning and prediction of the decisionmaking power of consumers. At last, it calculates and analyzes the two cases from the perspectives of network individual behavior and network group behavior and gives the comparison of two cases whether to consider irrational emotional factors. At the same time, it selects experts to evaluate the predicted results, tries to obtain a large number of data information of group consumers from different perspectives, and makes purchase decision-making reasoning, This paper attempts to further explore the group buying behavior of online consumers.

The main structure of this article is introduced as follows. The first part introduces the background and significance of the topic, as well as the work and organizational structure of this article. The second part introduces related work, as well as the concepts of set theory and fuzzy reasoning in fuzzy mathematics, as well as the more popular rational behavior theory in the field of behavior prediction. The third part takes mobile phone reviews as the experimental object to conduct data collection and experimental steps; the fourth part realizes the prediction of decision-making power, discusses the possibility of approximate reasoning of online group buying behavior, and gives examples to verify the rationality of the prediction algorithm. The fifth part is a summary of the full-text work.

\section{Proposed Method}

2.1. Related Work. Portfolio management includes deciding which assets to include in the portfolio based on the objectives of investors and changing market and economic conditions. The always difficult selection process involves determining which assets to purchase, how many assets to purchase. Chen proposed a new meme neural fuzzy system (MNFS-FPM) for financial portfolio management, which simulates the thinking process of rational investors and generates the optimal portfolio from a group of assets according to the selected investment style. The system is mainly composed of two modules: General self-organizing fuzzy neural network (GenSoFNN-Yager) to realize Yager reasoning, which is used to predict the expected return of each asset; memory algorithm of simplex local search (MA-NM/SMD) is used to determine the optimal investment weight distribution of each asset in the portfolio. The experimental results of the Dow Jones Industrial Average (DJIA) stock show that the system proposed in this paper has better performance than the existing statistical mean variance analysis and capital asset pricing model (CAPM) [1].

The future web business model involves a virtual environment, in which entities interact to sell or purchase information products. This environment is called the information market (IMS). The intelligent agent is used in IMS to represent the buyer or information provider (seller). Daniela Šálková focuses on the decisions made by the buyer in the procurement negotiations with the seller. A kind of reasoning mechanism based on fuzzy logic is put forward. The knowledge of the buyer negotiation process is modeled by a fuzzy set. They propose a fuzzy reasoning machine to deal with the decisions made by the buyer at each stage of the negotiation process. The results of the proposed reasoning method indicate whether the buyer should accept or reject the seller's offer. Their findings are very promising for the efficiency of automated transactions by intelligent agents [2].

The policy of purchasing chips is decided by the notebook computers and computers of the original equipment manufacturers (OEMs) through similarity standards and probability rules. Sax's research aims to establish an expert system for predicting the purchase behavior of the semiconductor market. Similarity criteria and probability rules are extracted from the OEM information sequence of the quarterly list of the semiconductor market. Through the statistical methods of data collection and data mining, the rules of OEM purchasing behavior data are analyzed and extracted and transformed into fuzzy sets. In addition to the information from the nature of the market, they also created an inference expert system. Their analysis of similar products shows that when purchasing similar products, there are mainly two categories of OEM that use probability rules for one-year recovery information processing, with an average score of about $95 \%$ per quarter [3].

\subsection{Basic Theory of Artificial Neural Network}

2.2.1. Artificial Neural Network. Artificial neural network (ANN) is an adaptive nonlinear dynamic system formed by standardized neuron connection. At present, it has been deeply explored in the world. Through the connection of many simple artificial neurons, the ability of a biological neural network is simulated [4]. From other neurons or the external environment, obtain the consultation, and simply operate on it, and output the results to other artificial neurons and the external environment [5]. Under the influence of input information, the neural network will enter into the corresponding state. Because of the dynamic characteristics of neurons and their connections with each other, this excitation mode will automatically change into a new equilibrium state. In this way, this kind of neural network can define the corresponding mode transformation and realize the mapping relationship [6].

Because in the network, the corresponding connection mode of an artificial neuron is different, that is to say, a variety of artificial neural network modes are formed. Among them, the most widely used and representative model is the BP network, which is an error back propagation network 
[7]. In practice, BP neural network model is widely used, which consists of input, output, and hidden layer. As far as the transfer function is concerned, most of them are based on the application of the sigmoid function, which can approach all continuous functions and has good nonlinear mapping ability. In addition, the related parameters, including the network learning coefficient, can be set according to the specific situation, so the flexibility is more prominent. Therefore, in many areas, the role of this model is particularly prominent. Relying on this technology, a nonlinear evaluation model can be constructed, which can properly solve the randomness of weight definition, and better ensure the accuracy and scientificity of evaluation results and activities $[8,9]$.

\subsubsection{Classification of Artificial Neural Network. According} to the topological structure of the network, the artificial neural network can be classified into prefeedback grid [10].

(1) Feedforward Network. Input the information flow from the input layer, and then transmit it to the lower layer. After the standardized network processing, output it from the output layer. In this process, there is no feedback flow. The whole structure of neurons arrangement is input layer, hidden layer, and output layer. For neural networks, they are usually multilayer feedforward networks [11]

(2) Feedback Network. It can combine the connection weight, set the output from the corresponding layer as the input, and feed back to the previous layer or the same layer, which is called feedback network. According to the classification of learning strategies, it can be divided into the following categories, namely, the most appropriate application network, association, supervision, and unsupervised learning [12]

2.2.3. Operation Process and Sample Classification of Artificial Neural Network. As far as the operation of the artificial neural network is concerned, it involves two stages, one is the recall stage, and the other is the learning process [13].

(1) The learning process is the process that the network learns the rules in the sample according to the learning algorithm, so as to adjust the network weight. There are three kinds of learning algorithms: association, supervised, and unsupervised. All algorithms are often obtained by deriving the energy function. In essence, this process is the process of minimizing energy function. Therefore, the learning effect of the network can be reasonably measured through the energy function $[14,15]$

(2) Recall process is a process that combines the use of a recall algorithm. The network uses input information to make the output information clear. It can be divided into three categories: association, supervision, and unsupervised recall. During the operation of artificial neural network, the learning information is generally sampled, which can be divided into the following three categories: training samples in the learning process, the network will often use the training samples, so as to adjust the weighted value of the network [16]. It can be divided into association, supervision, and unsupervised training samples. When the test samples complete the learning process and evaluate the network learning effect, they will use the test samples, which have the same type as the training samples. After the learning process, the samples to be pushed can use the network to calculate the target samples, which is different from the test samples

\subsection{Fuzzy Mathematics}

2.3.1. Fuzzy Phenomenon. The concept of the fuzzy phenomenon is a kind of phenomenon that the boundary division is not strict and cannot be described with an accurate scale. No matter in human society or in nature, there are a lot of fuzzy phenomena. The concept embodied in this phenomenon is the fuzzy concept. In terms of humanities, life sciences, etc., there are often the most concentrated fuzzy concepts $[17,18]$. For example, the phenomenon of rain, people's description of rainfall, often for heavy rain, drizzle, and so on [19]. However, the standard of heavy rain and drizzle is often hard to say, and there is no clear boundary, so it is considered as a fuzzy division. In addition, as for the characteristics of the same person, it can accurately describe the level of education, gender, etc., and all people have a clear gender, culture, etc. At the same time, there are many people's characteristics, which cannot be clearly described. For example [20], the human health status expressed by poor, good, etc. belongs to qualitative estimation, which belongs to a subjective judgment carried out by people themselves. In terms of daily life, there are relatively many such cases $[21,22]$. Fuzzy mathematics, a mathematical subject, is to explore and deal with fuzzy phenomena from the perspective of self-quantity. Classical sets are difficult to describe fuzzy concepts accurately, because it is difficult to distinguish them in a single way. For its conceptual level, it is not absolute 1 and 0 . Therefore, if we want to describe fuzzy concepts or phenomena quantitatively, we need to expand the classical set [23].

2.3.2. Fuzzy Sets. For a common set $\mathrm{A}$, any element $\mathrm{X}$ in the space, either $x \in A$ or $x \notin A$, must be one of them. This feature can be represented by a function:

$$
A(x)=\left\{\begin{array}{l}
1 x \in A, \\
0 x \notin A .
\end{array}\right.
$$

$A(x)$ is the characteristic function of set A. In this paper, the characteristic function is extended to the fuzzy set, and only two values of 0 and 1 are taken in the general set.

Definition 1. Sets $\mathrm{x}$ as the whole field. If a is a function with value $[0,1]$ on $X$, then a is called fuzzy set.

For example, if five students are graded on the stability degree of their personalities, they are graded on the basis of 
the percentage system and, then, divided by 100 , a mapping from the domain $x=\{x 1, X 2, X 3, x 4, X 5\}$ to the $[0,1]$ closed interval is given.

$$
\begin{gathered}
X_{1} 85, \text { is } A\left(x_{1}\right)=0.85 \\
X_{2} 75, A\left(x_{2}\right)=0.75, \\
X_{3} 98, A\left(x_{3}\right)=0.98, \\
X_{4} 30, A\left(x_{4}\right)=0.30 \\
X_{5} 60, A\left(x_{5}\right)=0.60 .
\end{gathered}
$$

In this way, a fuzzy subset $A=(0.85,0.75,0.98,0.30,0.60)$ is determined.

Define 2 if $\mathrm{A}$ the first mock exam on any $X$, and $A \lambda=$ $\{x \mid x \in X, A(x \geq \lambda)$ for any $0 \leq \lambda \leq 1, A \lambda$ is $A$ cut set. $A \lambda$ is a normal set rather than a fuzzy set. Because the boundary of the fuzzy set is fuzzy, if we want to transform a fuzzy concept into mathematical language, we need to select different confidence level $\lambda(0 \leq \lambda \leq 1)$ to determine its membership relationship [24]. $\lambda$ cut set is the method of transforming the fuzzy set into the common set. Fuzzy set A is a set with wandering boundary, which increases with the decrease of $\lambda$ value, that is, when $\lambda_{1}<\lambda_{2}$, there is $\mathrm{A} \lambda_{1} \cap \mathrm{A} \lambda_{2}$.

Definition 2. Definition of fuzzy set operation. If A and B are two fuzzy sets on $\mathrm{X}$, their sum set, intersection set and A's remainder set are all fuzzy sets, and their membership functions are defined as follows:

$$
\begin{gathered}
(A \vee B)(x)=\max (A(x), B(x)), \\
(A \wedge B)(x)=\min (A(x), B(x)), \\
A^{C}(x)=1-(A(x) .
\end{gathered}
$$

The sum and intersection operations of fuzzy sets can be extended to any number of fuzzy sets [25].

2.3.3. Fuzzy Comprehensive Evaluation. A comprehensive evaluation is to make a general evaluation of the things or objects restricted by many factors, which is a common problem in daily life and scientific research work, such as product quality evaluation, scientific and technological achievements appraisal, and evaluation of a crop planting adaptability, all belong to the comprehensive evaluation problem. Due to the fuzziness and subjectivity in the evaluation of things from many aspects, the comprehensive evaluation with the fuzzy mathematics method will make the results as objective as possible and achieve better practical results [26].

The mathematical model of fuzzy comprehensive evaluation can be divided into one level model and multilevel model. Generally, it can be concluded into the following steps:

(1) Establish the factor set $U=\left\{u_{1}, u_{2}, \cdots, u_{n}\right\}$. Factors are all kinds of attributes or performance of the object. In different situations, they are also called parameter indexes or quality indexes. They can com- prehensively reflect the quality of the object, so they can be used to evaluate the object [27]

(2) Establish evaluation set $V=\left\{v_{1}, v_{2}, \cdots, v_{n}\right\}$. For example, for the evaluation of industrial products, the evaluation set is the set of grades, and the evaluation set is the set of adaptability

(3) To establish a single factor evaluation is to establish a fuzzy mapping from u to $\mathrm{F}(\mathrm{V})$ :

$$
f: U \longrightarrow F(V), \forall u_{i} \in U
$$

The fuzzy relation can be induced by F, and the fuzzy matrix can be obtained

$$
R=\left[\begin{array}{ccc}
r_{11} r_{12} & \cdots & r_{1 m} \\
r_{21} r_{22} & \cdots & r_{2 m} \\
\cdots \cdots & \cdots & \cdots \\
r_{n 1} r_{n 2} & \cdots & r_{n m}
\end{array}\right] .
$$

$R$ is called a single factor evaluation matrix, so $(U, V, R)$ constitutes a comprehensive evaluation model.

(4) Comprehensive Evaluation. Due to the different emphasis on each factor in $U$, each factor needs to be given different weights, which can be expressed as a fuzzy subset $A=\left(a_{1}, a_{2}, \cdots, a_{n}\right)$

After $R$ and $A$ are calculated, the comprehensive evaluation model is $B=A o R$. Note $B=\left(b_{1}, b_{2}, \cdots, b_{m}\right)$, which is a fuzzy subset of $V$, where

$$
b_{j}=\bigvee_{i=1}^{n}\left(a_{i} \wedge r_{i j}\right)(j=1,2, \cdots, m)
$$

If the result is $\sum_{j=1}^{m} b_{j} \neq 1$, the result will be normalized.

From the above four steps of a fuzzy comprehensive evaluation, it can be seen that the establishment of single factor evaluation matrix $\mathrm{R}$ and the determination of weight distribution a are two key tasks, but at the same time, there is no unified format to follow, which can generally be obtained by statistical experiments or expert scoring methods $[28,29]$.

2.3.4. Semantic Fuzziness of Online Comments. Online reviews mainly include two categories: cognitive words and emotional words. Cognitive and emotional words are very broad concepts with fuzzy attributes. Taking emotion as an example, the current emotional semantic analysis is still in its infancy, and the classification of emotion is relatively rough, which is only based on the dual tendency analysis of "yes" and "no." Emotion has uncertainty, which makes it difficult to deal with it by simple quantitative means, so we choose the method of fuzzy set to build the semantic fuzzy system of multi sentiment analysis [30].

The emotional words and cognitive words in online reviews are fuzzified, and the positive and negative degree 


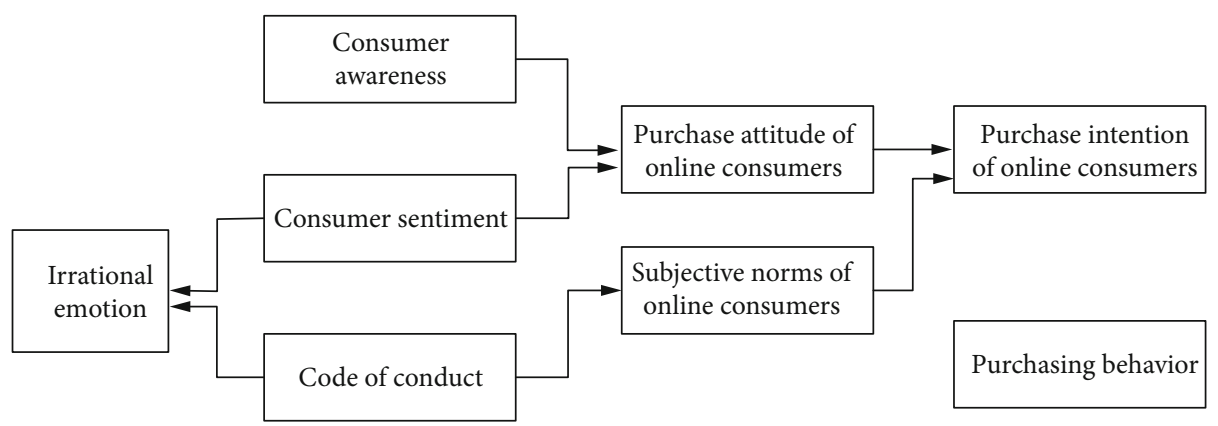

FIGURE 1: Theoretical framework of behavior prediction.

measures are, respectively, divided into levels: each level corresponds to a fuzzy membership function, $\mathrm{s}$ (less), $\mathrm{m}$ (medium), $\mathrm{I}$ (large), V (very), and Z (none) without polarity, as shown in the formula.

$$
P(w)=P_{w}\left(x, \sigma_{w}, c_{w}\right)=\exp \left[\frac{-\left(x-c_{w}\right)^{2}}{2 \sigma_{w}^{2}}\right]
$$

Among them, $w$ represents the emotion level of vocabulary, $w \in\{-V L,-L,-M,-S, Z,+S,+M,+L,+V L\}, \sigma_{w}, c_{w}$ are the expectation and standard deviation of Gaussian membership function of a corresponding level, $x \in[-a, a]$ represents the emotion value of consumers, and a is a real number greater than zero (representing the boundary of emotion domain).

(5) The construction of consumer decision-making power model with rational and irrational consideration

Based on the current theoretical development and the redefinition of the connotation and extension of the constituent variables of the existing models in the field of online purchase, a prediction model of online purchase behavior with rational and irrational consideration is proposed as shown in Figure 1. This kind of network irrational emotion is mostly expressed through network comments on the evaluation of the network products or services after purchase and will directly affect the purchase attitude of other network consumers, and then affect their purchase behavior.

2.4. Fusion of Artificial Neural Network and Fuzzy Mathematics. According to the form and function of connecting the two, the combined form of the model can be summarized as follows:

Series type: as for the system, the two are connected through the application of series mode, i.e., output and input correspondence. Neural network and fuzzy system can be used as input and output, respectively, and vice versa.

Parallel type: in terms of the system, the two are connected through the use of parallel mode, i.e., the input is the same. As far as neural networks are concerned, learning data can be used to describe the range of network characteristics as a workspace. In addition, it is necessary to use the
TABLE 1: Mamdani reasoning.

\begin{tabular}{lcc}
\hline Attitude & Norm & Behavior intention \\
\hline 1.88 & 0.72 & 5.72 \\
\hline
\end{tabular}

fuzzy function to calculate the output value in the extreme and boundary region.

Network learning type: the fuzzy system is the system. Combining with the neural network system, i.e., control rules, the output and input information of this process can be combined to carry out adaptive adjustment. On the contrary, the connection of neurons can be adjusted through the use of fuzzy logic.

Structural equivalence: the equivalent structural neural network can be used to represent the fuzzy system. For neural network, all parameters and nodes have a very clear meaning. It is not a black box structure, but it does not cover the obvious dividing line in the combination.

\section{Experiments}

3.1. Data Acquisition. The corpus in this case comes from https://taobao.com. In order to predict the decision-making power of network consumers, we choose two products C1 and C2 (Note: C1 Samsung i9103, C2 Samsung i9105, two Samsung phones have similar function and performance). More than 190 evaluation records were downloaded through the web statement collection tool "network spirit". The repeated and worthless comments were removed, and 188 effective comments were finally selected as the comment set A. Select a user who has browsed a mobile phone but has not purchased it. In the same way, 98 effective comments are finally selected and set as comment set B.

In other words, the values of "attitude" and "norm" are used as forward language changes, and the constructed rules are used to infer the subsequent parts. According to the comprehensive emotion fuzzy calculation algorithm, combined with example calculations in the previous chapter, we will evaluate the attitudes corresponding to the $\mathrm{B}$ set and the subjective criteria corresponding to the $\mathrm{A}$ set as the reason for the fuzzy calculation.

3.2. Research Object. This paper focuses on the decisionmaking power of online consumers. The measurement of behavioral intention is mostly based on the measurement table. In the development process of the measurement table, 


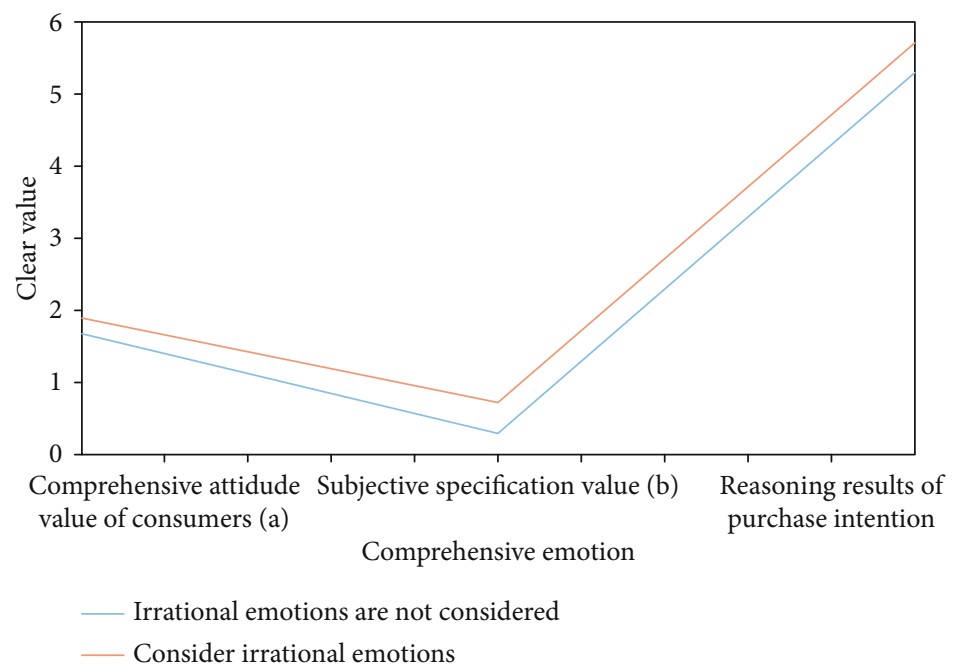

Figure 2: Fuzzy inference results of individual behavior intention.

different levels of scales will be used to measure the decisionmaking power of consumers, and the table will be adjusted appropriately according to the actual situation. However, due to the uncertain psychological variables such as emotion, cognition, and attitude, which cause the decision-making power of online consumers, it is difficult to clearly describe the fuzzy attribute of purchase behavior, so the fuzzy set method can be used to classify. The decision-making power of online consumers is divided into seven levels from weak to strong. The degree of decision-making power of all online consumers is between the extreme tendency and the extreme nontendency.

3.3. Calculation Method. Based on the consumer information behavior model in consumer behavior, Mamdani-type fuzzy inference is used to formulate fuzzy inference rules under different weights. The principle is that attitude variables dominate, and subjective normative variables play a secondary role. $R_{c}\left(A_{1}, A_{2}\right)=\int \vee\left(\mu_{A_{1}}\left(u_{1}\right) \wedge \mu_{A_{2}}\left(u_{2}\right), 1-\mu_{A_{1}}\left(u_{1}\right)\right) /\left(u_{1}\right.$, $\left.u_{2}\right)$ is used to determine the compound relationship of rules in the case of two antecedents. In this fuzzy reasoning system, some basic attributes are set as follows: "and" calculation and fuzzy implication adopt minimum operation and "or" calculation and fuzzy rule synthesis adopt maximum operation.

\section{Discussion}

4.1. Reasoning and Prediction of Decision-Making Power of Individual Consumers on the Internet. According to the steps of the experiment, we get the comprehensive emotion value and the upper norm value under the condition of considering irrational emotion, i.e., attitude $=1.88$ and norm $=0.72$. According to this, we can directly deduce the "behavior intention" value of the consumer. The reasoning is shown in Table 1 . We can get behaviorintention $=5.72$ through Mamdani reasoning, and we can get behavior intention = 5.72 through fuzzy set processing. Finally, it is determined that the consumer's purchase intention for the product is close to the VT mode, which is "very inclined." Each line of the reasoning process diagram is represented as a reasoning rule. Each rule antecedent is small, then mapped to the reasoning consequent, and then, all the reasoning consequent is large. Finally, the aggregate output membership function is obtained, and the behavior intention value is finally obtained by using the centroid method.

In the same way, we skip the consideration step of irrational emotion factors in the comprehensive emotion fuzzy calculation method which takes both rational and irrational into account. The purpose of neglecting this step is to try to conduct behavioral intention reasoning without considering irrational emotion factors, so as to make an effective comparison between them. Based on the same comment set and the same reasoning method, the clear values of the two methods are finally obtained as shown in Figure 2.

Through fuzzy set processing, it is finally determined that without considering irrational emotional factors, it can be seen that considering irrational emotional factors has a significant impact on online shopping consumer behavior intentions, and consumers' decision-making power on products is close to the PT model. The consumers are more affected by the positive irrational emotion, and their shopping behavior intention mode deviates to a certain extent, so they are inclined consumers.

4.2. Reasoning and Prediction of Decision-Making Power of Consumers in Network Groups. For product C1, we further select 51 more representative online consumers, whose oral characteristics of online comments are obvious, shopping time and scope are more extensive, and they can represent online shopping groups. Considering the comprehensive emotional factors of each consumer, the same method is used to calculate, and relevant statistics are carried out to get their purchase tendency for the product. For C2, we also select a certain amount of potential network consumption and use the same method to get their purchase tendency for this product, as shown in Figure 3.

By comparison, it can be seen that the group buying intention of $\mathrm{C} 1$ products is close to the VT mode, indicating 


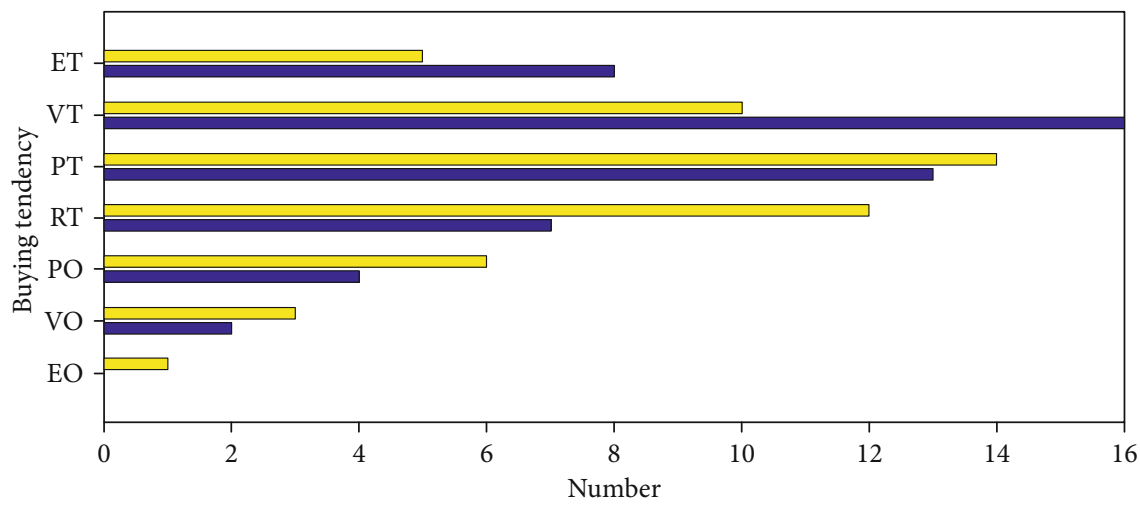

$\square \mathrm{C} 1$

$\mathrm{C} 2$

FIgURE 3: Distribution of online group purchase intention patterns.

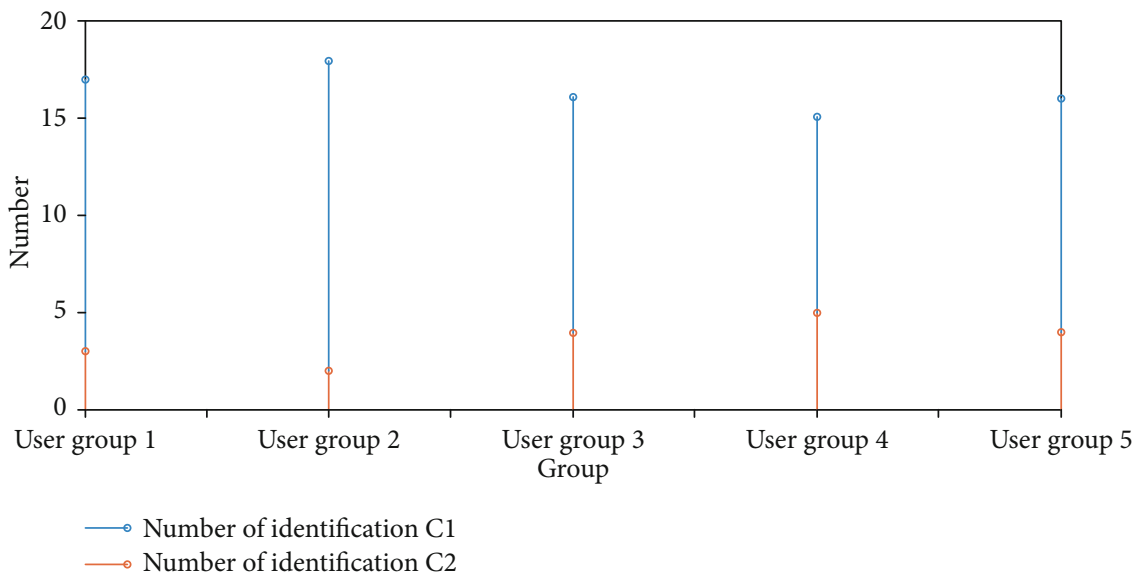

FIGURE 4: Statistical results of user group identity.

that the group buying intention of $\mathrm{C} 2$ products is close to the $\mathrm{RT}$ mode, and that the group buying intention of $\mathrm{C} 1$ products is relatively average. As a similar product, $\mathrm{C} 1$ shows a higher purchase intention than $\mathrm{C} 2$, indicating that $\mathrm{C} 1$ produces more various purchase factors and is a more attractive commodity. Therefore, most buyers prefer $\mathrm{C} 1$ when they choose between $\mathrm{C} 1$ and $\mathrm{C} 2$.

For the results of this fuzzy inference prediction, five groups of users who are familiar with this kind of mobile phone products participated in the evaluation process of the system prediction effect, each group of 20 people, each of them made a click choice according to their own needs and understanding of the product. The evaluation of these five user groups is equivalent to the experts of the industry, and the experts' guidance for consumers through evaluation is equivalent to fuzzy prediction, which shows the intelligence. If the user group agrees with the predicted result, it indicates that the prediction is successful; otherwise, it indicates failure. The statistical result is shown in Figure 4.

The number of identification here indicates that the prediction results of the system are consistent with the opinions of experts, while the number of disagreement indicates that the prediction results are inconsistent with the opinions of
TABLE 2: Statistical results of user group identification.

\begin{tabular}{lcc}
\hline User group & $\begin{array}{c}\text { Number of } \\
\text { identification C1 }\end{array}$ & $\begin{array}{c}\text { Number of } \\
\text { identification C2 }\end{array}$ \\
\hline User group 1 & 10 & 10 \\
User group 2 & 12 & 8 \\
User group 3 & 9 & 11 \\
User group 4 & 13 & 7 \\
User group 5 & 11 & 9 \\
\hline
\end{tabular}

experts. In the absence of online comment data from Internet users, online shopping malls generally take sales volume and user rating as their opinions, which is reasonable and ignores the emotional needs of consumers. In order to simulate this traditional method, five groups of experts were asked to take the product sales volume, average level of play, and the number of user comments as the basis. Similarly, five-user groups click on two products, respectively, 20 people in each group, 20 times in total. The statistical results are shown in Table 2.

In Figure 5, the user's recognition of product $\mathrm{C} 1$ reaches $82 \%$, while in Table 2, the user's recognition is $55 \%$. The comparison of the two methods fully conforms to the 


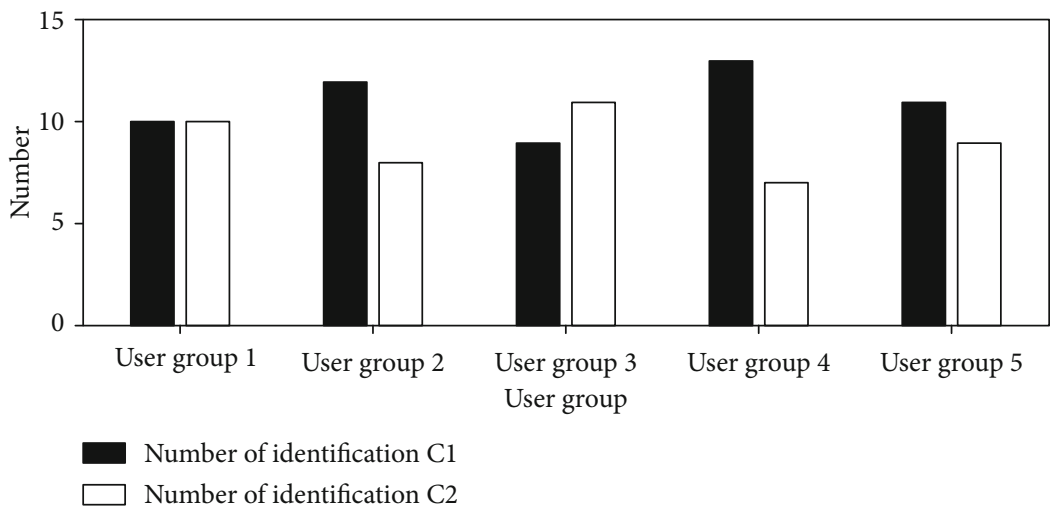

Figure 5: Statistical results of user group identification.

expectation in advance. The first method digs out the user's emotion and evaluation information from the comments, fully considers the personalized needs of the consumers, so it is closer to the prediction results of the system. The second method essentially recommends from the attention degree of the products and the overall quality of the products, less considers the comment information of the consumers. Therefore, there is a deviation from the predicted results.

\section{Conclusions}

This paper extracts consumers' online comments, uses the fuzzy attributes of natural language, calculates consumers' cognitive and emotional information with semantic fuzzy, fully considers the irrational emotional factors in online shopping, and innovatively expands rational behavior. Model and propose reasonable and irrational behavior prediction models. At the same time, the fuzzy semantic modeling is used to transfer and transform the membership function of emotional words, and a comprehensive emotional calculation method of combining rationality and irrationality is put forward innovatively. The fuzzy reasoning rules are constructed based on the subjective attitude and behavior norms of consumers in the extended model. Realize the reasoning of online buyers' purchase intention.

Finally, through case-based reasoning calculation, the distribution of three comprehensive emotional degrees of individuals and group products is calculated, and it is concluded that the comprehensive emotional degree of the group approximately obeys the normal distribution, and the average is in a more rational level. Furthermore, the reasoning of individual behavior of online consumers verifies the significant influence of considering irrational emotional factors on online shopping behavior intention. At last, the object is extended to the network purchasing group to realize the approximate reasoning of consumer group behavior. In the method in this paper, the user's recognition rate of product $\mathrm{C} 1$ is $82 \%$. The method in this paper extracts the user's emotion and evaluation information from the comments, fully considers the individual needs of consumers, and is closer to the system's prediction results.

There are two main innovations in this paper. One is to make full use of the fuzzy semantic resources in online reviews, to fully consider the irrational emotions, to put forward a behavior prediction model combining rationality and irrationality, and to give a comprehensive emotion calculation method combining the two. The second is to use the fuzzy inference system to predict the individual consumption behavior of the network and to explore the group consumption behavior of the network, which greatly enriches the traditional theory and research methods of network consumption prediction.

\section{Data Availability}

Data studies are not covered in the article.

\section{Conflicts of Interest}

The authors declare that they have no conflicts of interest.

\section{References}

[1] Y. Chen, W. Wei, and F. Liu, "CES utility function based consumer optimal decision making in heat-power market," Dianli Xitong ZidonghualAutomation of Electric Power Systems, vol. 42, no. 13, pp. 118-126, 2018.

[2] D. Šálková and A. Hes, "Gluten-free food-the influence of selected qualitative characteristics on consumer decision making of coeliacs in hospitality establishments," Czech Journal of Food Sciences, vol. 33, no. 6, pp. 513-517, 2015.

[3] J. K. Sax, "Biotechnology and consumer decision-making," Seton Hall Law Review, vol. 47, no. 2, p. 433, 2017.

[4] D. O'Rourke and A. Ringer, "The impact of sustainability information on consumer decision making," Journal of Industrial Ecology, vol. 20, no. 4, pp. 882-892, 2016.

[5] F. Rothenfluh, E. Germeni, and P. J. Schulz, "Consumer decision-making based on review websites: are there differences between choosing a hotel and choosing a physician?," Journal of Medical Internet Research, vol. 18, no. 6, article e129, 2016.

[6] A. Kumar, A. Vohra, and H. K. Dangi, "Consumer decisionmaking styles and post purchase behaviour of poor for Fast Moving Consumer Goods," International Journal of Consumer Studies, vol. 41, no. 2, pp. 121-137, 2017.

[7] E. B. H. Treichler and D. William, "Beyond shared decisionmaking: collaboration in the age of recovery from serious 
mental illness," The American Journal of Orthopsychiatry, vol. 87, no. 5, pp. 567-574, 2017.

[8] Y. Joshi and Z. Rahman, "Predictors of young consumer's green purchase behaviour," Management of Environmental Quality An International Journal, vol. 27, no. 4, pp. 452-472, 2016.

[9] A. Hortaçsu, S. A. Madanizadeh, and S. L. Puller, "Power to choose? An analysis of consumer inertia in the residential electricity market," Nber Working Papers, vol. 9, no. 4, pp. 192-226, 2017.

[10] R. Babcock, "Medical decision-making for minors: using care ethics to empower adolescents and amend the current power imbalances," Asian Bioethics Review, vol. 8, no. 1, pp. 4-19, 2016.

[11] M. Zubatsky, "Relationship power in health care: science of behavior change, decision making, and clinician self-care," Family Medicine, vol. 50, no. 1, pp. 70-71, 2018.

[12] Y. Bao, C. Feng, and T. Xu, "Online security and stability comprehensive auxiliary decision-making of power system," Automation of Electric Power Systems, vol. 39, no. 1, pp. 104110, 2015.

[13] T. Zhu, J. Ding, and X. Zheng, "A comprehensive decisionmaking method for power network planning schemes based on the combination of the improved TOPSIS method with Delphi-entropy weight method," Power System Protection \& Control, vol. 46, no. 12, pp. 91-99, 2018.

[14] S. L. Lima, O. R. Saavedra, and V. Miranda, "A two-level framework to fault diagnosis and decision making for power transformers," IEEE Transactions on Power Delivery, vol. 30, no. 1, pp. 497-504, 2015.

[15] J. Sousa, O. R. Saavedra, and S. L. Lima, "Decision-making in emergency operation for power transformers with regard to risks and interruptible load contracts," IEEE Transactions on Power Delivery, vol. 33, pp. 1556-1564, 2017.

[16] P. P. Zubcsek, Z. Katona, and M. Sarvary, "Predicting mobile advertising response using consumer co-location networks," Journal of Marketing, vol. 81, no. 4, pp. 109-126, 2017.

[17] Y. Liu, H. Li, X. Xu, V. Kostakos, and J. Heikkilä, "Modeling consumer switching behavior in social network games by exploring consumer cognitive dissonance and change experience," Industrial Management \& Data Systems, vol. 116, no. 4, pp. 801-820, 2016.

[18] S. Ding, S. Qu, Y. Xi, and S. Wan, "Stimulus-driven and concept-driven analysis for image caption generation," Neurocomputing, vol. 398, 2020.

[19] W. H. Allen, A. Rubaai, and R. Chawla, "Fuzzy neural network-based health monitoring for HVAC system variable-air-volume unit," IEEE Transactions on Industry Applications, vol. 52, no. 3, pp. 2513-2524, 2016.

[20] J.-Y. Yeh and C.-H. Chen, "A machine learning approach to predict the success of crowdfunding fintech project," Journal of Enterprise Information Management, 2019.

[21] M. Billah and S. Waheed, "Predicting closing stock price using artificial neural network and adaptive neuro fuzzy inference system (ANFIS): the case of the dhaka stock exchange," International Journal of Computer Applications, vol. 129, no. 11, pp. 1-5, 2015.

[22] S.-Y. Chen, C.-Y. Lee, C. H. Wu, and Y. H. Hung, "Intelligent motion control of voice coil motor using PID-based fuzzy neural network with optimized membership function," Engineering Computations, vol. 33, no. 8, pp. 2302-2319, 2016.
[23] M.-L. Zhou, J.-A. Zhang, and Y. Zhao, "Hybrid control for piezoelectric micro positioning platform based on BP neural network and expert fuzzy control," Control \& Decision, vol. 33, no. 1, pp. 95-100, 2018.

[24] Y. Zhang and Q. Gao, "Comprehensive prediction model of water quality based on grey model and fuzzy neural network," Chinese Journal of Environmental Engineering, vol. 9, no. 2, pp. 537-545, 2015.

[25] M. Elhoseny, G.-B. Bian, S. K. Lakshmanaprabu, K. Shankar, A. K. Singh, and W. Wu, "Effective features to classify ovarian cancer data in internet of medical things," Computer Networks, vol. 159, pp. 147-156, 2019.

[26] Y. Deng, Z. Ren, Y. Kong, F. Bao, and Q. Dai, “A hierarchical fused fuzzy deep neural network for data classification," IEEE Transactions on Fuzzy Systems, vol. 25, pp. 1006-1012, 2017.

[27] S. Azali and M. Sheikhan, "Intelligent control of photovoltaic system using BPSO-GSA-optimized neural network and fuzzy-based PID for maximum power point tracking," Applied Intelligence, vol. 44, no. 1, pp. 88-110, 2016.

[28] Z. Lv, S. Zhang, and W. Xiu, "Solving the security problem of intelligent transportation system with deep learning," IEEE Transactions on Intelligent Transportation Systems, vol. 22, pp. 4281-4290, 2021.

[29] X. Xu, D. Cao, Y. Zhou, and J. Gao, "Application of neural network algorithm in fault diagnosis of mechanical intelligence," Mechanical Systems and Signal Processing, vol. 141, article 106625, 2020.

[30] S. K. Paul, A. Azeem, and A. K. Ghosh, "Application of adaptive neuro-fuzzy inference system and artificial neural network in inventory level forecasting," International Journal of Business Information Systems, vol. 18, no. 3, pp. 268-284, 2015. 